\title{
Vehicle Identification Using Wavelet Entropy and Particle Swarm Optimization Support Vector Machine
}

\author{
Fangzhou Bao ${ }^{1}$, Koji Nakamura ${ }^{2}$ \\ ${ }^{1}$ School of Computer Science and Technology, Nanjing Normal University, Nanjing, Jiangsu 210023, China \\ ${ }^{2}$ School of Computer Science and Systems Engineering, Kyushu Institute of Technology, Iizuka-shi, \\ Fukuoka Prefecture 820-8502, Japan
}

Keywords: Wavelet Entropy, Particle Swarm Optimization, Support Vector Machine, Vehicle Identification

\begin{abstract}
In order to identify Ford vehicles from non-Ford vehicles, this paper proposed a novel method based on the combination of wavelet entropy, particle swarm optimization, and support vector machine. We collect a 100-image dataset, 50 are Ford vehicles and the rest 50 are non-Ford vehicles. The results show that our method obtained a sensitivity of $82.20 \pm 3.94 \%$, a specificity of $81.60 \pm 3.50 \%$, and an accuracy of $81.90 \pm 0.74 \%$. In all, this method is promising in vehicle identification.
\end{abstract}

\section{Introduction}

With the rapid growth of the number of vehicles, the demand of vehicle production line supervision, traffic video surveillance is increasing $[\underline{1}, \underline{2}]$. As a result, the accuracy in vehicle identification is required to be getting higher. The core mission is to identify and classify vehicle in images $[\underline{3}, \underline{4}]$.

In this study, we used a model mixed by wavelet entropy and support vector machine based on particle swarm optimization (PSO-SVM). By applying wavelet entropy [5-9], we can extract a better expression of image signal from original image, which will be viewed as features of the image. As a result, extracted features will be the inputs of PSO-SVM. In the end, PSO-SVM will output the correct result of this task. According to out experiments, this method can provide both high accuracy and high speed, which makes it an advantage compared with neural networks.

With the perspective of practical application, we collect images of vehicles from multiple directions, instead of images of frontal view only. The cross validation technique [10] was used to report the out-of-sample errors.

\section{Method}

\subsection{Wavelet Entropy}

Information entropy is defined as the average amount of information produced by a stochastic source of data. We measure the information entropy of signals applied wavelet transform, which is called wavelet entropy (WE) [11-17].

The wavelet is a smooth and quickly vanishing oscillating function with good localization in both frequency and time [18]. A wavelet family $w f_{a, b}$ is the set of elementary functions generated by dilations and translations of a unique admissible mother wavelet $w f(t)$ :

$$
w f_{a, b}(t)=\frac{1}{\sqrt{|a|}} * w f\left(\frac{t-b}{a}\right)
$$

where $a, b \in R, a \neq 0$ are the scale and translation parameters respectively, and $\mathrm{t}$ is the time.

Assuming the signal sequence is $S(n)(n=0,1, \ldots, M), N=\log _{2} M$. Then the wavelet coefficients are given by:

$$
C_{j}(k)=<S, w f_{j, k}>
$$


The energy at each resolution level $j=-1, \ldots,-N$, will be the energy of the detail signal and the energy at each sampled time $\mathrm{k}$ will be:

$$
E_{j}=\sum_{j=-N}^{-1}\left|C_{j}(k)\right|^{2}
$$

As a result, the total energy can be calculated by:

$$
E_{\text {total }}=\sum_{j<0} E_{j}
$$

We can use $p_{j}$ to represent the relative wavelet energy:

$$
p_{j}=\frac{E_{j}}{E_{\text {total }}}
$$

In the end, we can obtain the wavelet entropy [19-23] based on the equation obtained in (5):

$$
W E=-\sum_{j<0} p_{j} * \ln \left[p_{j}\right]
$$

\subsection{Particle Swarm Optimization}

Particle swarm optimization (PSO) is an evolutionary algorithm that optimizes (minimizes) an objective function by iteratively trying to improve a set of candidate solution with regard to a given fitness metric [24]. It solves a problem by having a population of candidate solutions, here dubbed particles, and moving these particles around in the solution space according to simple mathematical formula over the particle's position and velocity. Each particle's movement is influenced by its local best known position, but is also guided toward the best known positions in the solution space, which are updated as better positions are found by other particles. This is expected to move the swarm toward the best solutions.

PSO starts from the random solution and iteratively finds the optimal solution. It also evaluates the quality of the solution through fitness, but it is simpler than the genetic algorithm rules. It does not have the "crossover" and "mutation" operations of the genetic algorithm. It seeks the global optimum by following the current searched optimal value [25-30].

PSO simulates birds by designing massless particles. Each particle has 2 properties: velocity $(V)$ and position $(P)$, where $V$ represents how fast it can move and $\mathrm{P}$ indicates where the particle is in the solution space. Each particle searches global optimum in the solution space. The best position experienced by $i$-th particle will be saved as pbest $_{i}$. Fitness of each particle will be shared among all particles. And the global optimum will be saved as gbest. Normally, $d$-th component of the solution vector will be clipped in the range $\left[x^{d}{ }_{\min }, x^{d}{ }_{\max }\right]$.

Then, we can use following formula to update particle's velocity:

$$
v_{i}^{d}=w * v_{i}^{d}+c_{1} * r_{1} *\left(\text { pbest }_{i}-x_{i}^{d}\right)+c_{2} * r_{2} *\left(\text { gbest }_{d}-x_{i}^{d}\right)
$$

And we use following formula to update particle's position:

$$
x_{i}^{d}=x_{i}^{d}+v_{i}^{d}
$$

where $c_{1}$, and $c_{2}$ are learning rate of PSO and $r_{1}, r_{2} \sim \mathrm{U}[0,1], \mathrm{v}_{\mathrm{i}}^{\mathrm{d}}$ represents $d$-th component of $i$-th particle's velocity, $x_{i}{ }^{d}$ represents $d$-th component of $i$-th particle's position.

After initialization, PSO will iteratively update velocity and position of each particle and save pbest and gbest, until satisfies the termination condition. In the end, gbest will be the solution of given problem. Due to page limit, we shall compare PSO with latest bioinspired algorithms, e.g., biogeography-based optimization [31-34], ant colony optimization, multiverse optimizer, etc.

\subsection{Support Vector Machine}

Support vector machine (SVM) which is also called large margin classifier is a model which can classify samples into 2 categories.

The set of points closest to the maximum margin decision boundary are known collectively as the support vectors [35]. The name comes from the fact that they define, or support, the decision boundary. In many situations, this will make the decision become a function of only a small subset of the training samples. For problems with large dataset, this can be a very useful feature. Consider classifying a test point using KNN when the training set containing a large number of objects. To 
find the set of neighbors, distances must be computed between the sample and all of the training objects. For an SVM trained on the same data, the decision function might just involve a much smaller subset of the training data.

When classification, we use following formula to determine the label of given sample $x_{n}$ :

$$
\text { label }=\left\{\begin{array}{c}
1, \text { if } w^{T} * x_{n}>1 \\
-1, \text { if } w^{T} * x_{n}<-1
\end{array}\right.
$$

where $\mathrm{w}$ is the weight vector which represents the normal vector of the decision boundary. Here we use PSO algorithm to find best $w$, instead of using some traditional algorithm such as SMO [36-38].

Original SVM can only classify samples which are linear separable. While, kernel functions such as Gaussian Kernel make it able to classify samples with non-convex decision boundary.

\subsection{Dataset and Implementation}

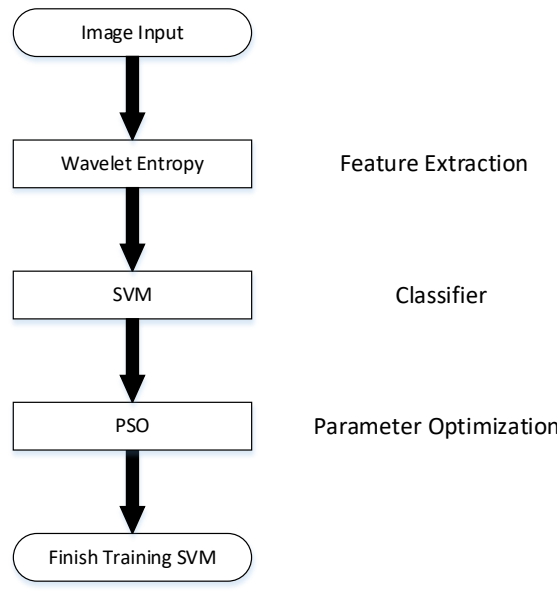

(a) Training

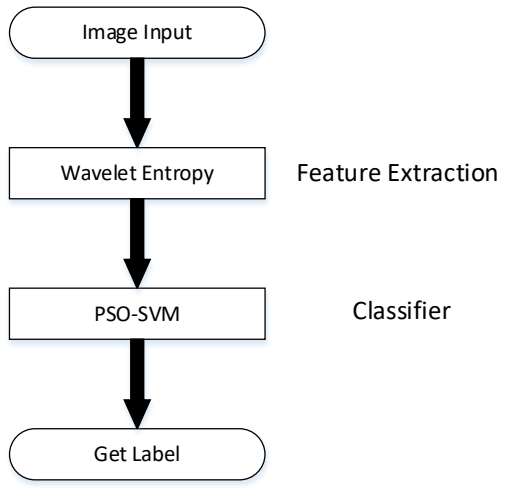

(b) Test

Figure 1 Pipeline of our method

In this study, we calculate the wavelet entropy of preprocessed input image. This entropy will be viewed as extracted features of input image and sent to SVM. Apparently features extracted by wavelet entropy are better than raw pixels of input image. PSO algorithm will be used to optimize parameters of SVM during training. We train SVM using mini-batches, which can save some training time. Performance of our algorithm will be shown in later Section.

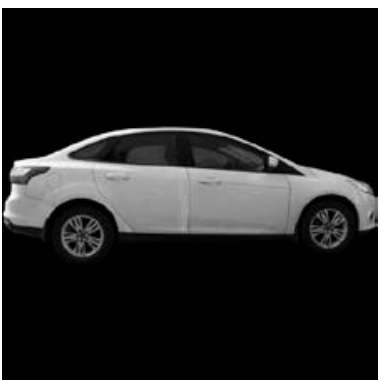

(a) Ford

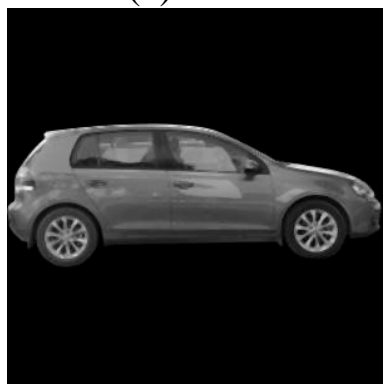

(d) Shanghai volks

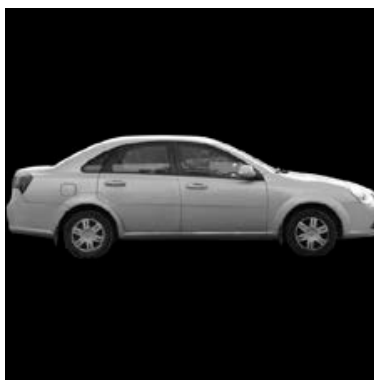

(b) Buick

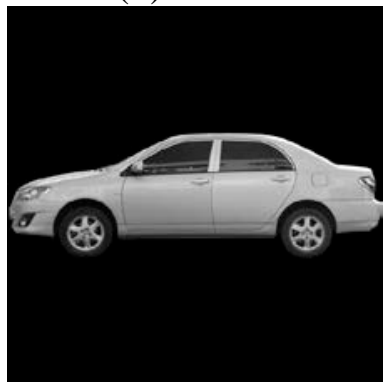

(e) Toyota

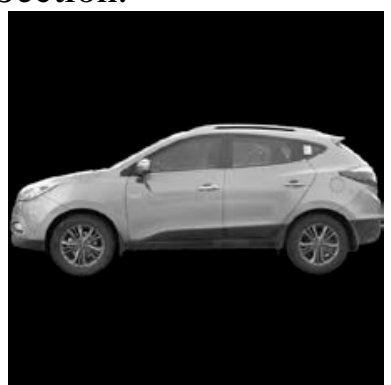

(c) Hyundai

Figure 2 Samples of Dataset 
100 vehicle images are obtained, 50 are Ford vehicles, and the rest include Buick, Hyundai, Shanghai Volks, and Toyota. Each image was resized to $256 \times 256$. For simplicity, we converted the color images to gray-level images.

\section{Experiments and Results}

\subsection{Cross Validation Techniques}

Here we use our proposed 'WE-PSOSVM' approach to identify Ford vehicle from other categories of vehicles. The parameters are obtained by grid searching approach. We used a four-level wavelet entropy decomposition. A 10x10-fold cross validation was used, since our dataset merely contains 100 image. One example of 10-fold division was shown in Figure 3.

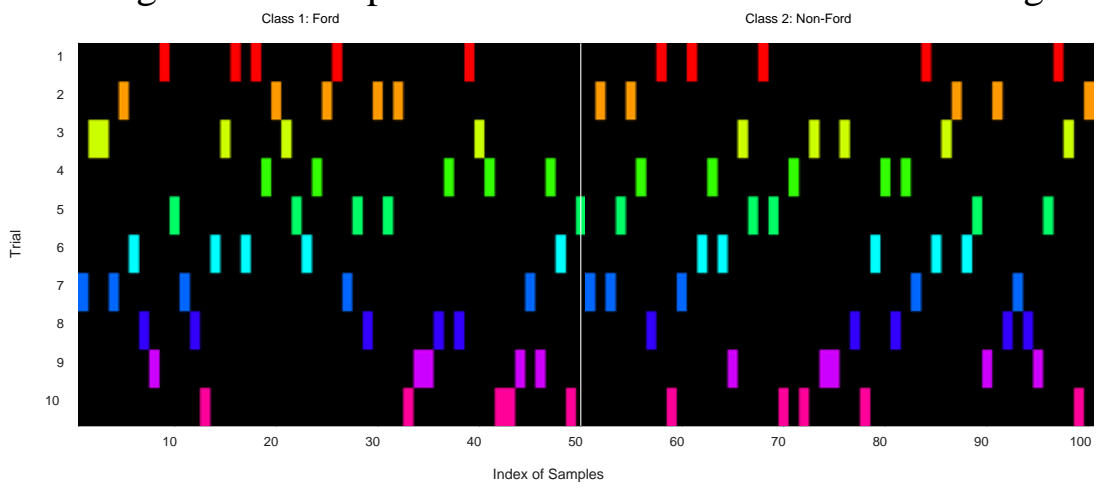

Figure 3 Division of ten folds

Table 1 Statistical Results of proposed methods

\begin{tabular}{|c|c|c|c|c|c|c|c|c|c|c|c|}
\hline Sen & $\mathrm{F} 1$ & F2 & F3 & F4 & F5 & F6 & F7 & F8 & F9 & F10 & Total \\
\hline 1 & 60 & 80 & 60 & 40 & 100 & 80 & 100 & 100 & 60 & 80 & 76 \\
\hline 2 & 100 & 40 & 80 & 80 & 60 & 80 & 80 & 100 & 100 & 100 & 82 \\
\hline 3 & 60 & 100 & 60 & 80 & 80 & 80 & 80 & 80 & 100 & 60 & 78 \\
\hline 4 & 80 & 100 & 80 & 100 & 80 & 80 & 60 & 80 & 80 & 80 & 82 \\
\hline 5 & 100 & 80 & 80 & 100 & 100 & 80 & 80 & 60 & 40 & 100 & 82 \\
\hline 6 & 100 & 100 & 100 & 100 & 80 & 80 & 80 & 80 & 100 & 80 & 90 \\
\hline 7 & 60 & 100 & 100 & 100 & 80 & 60 & 60 & 100 & 80 & 100 & 84 \\
\hline 8 & 60 & 80 & 20 & 100 & 100 & 100 & 100 & 100 & 100 & 100 & 86 \\
\hline 9 & 100 & 60 & 60 & 100 & 60 & 100 & 100 & 100 & 60 & 80 & 82 \\
\hline 10 & 80 & 80 & 100 & 60 & 100 & 100 & 100 & 80 & 40 & 60 & 80 \\
\hline Spc & F1 & F2 & F3 & F4 & F5 & F6 & F7 & F8 & F9 & F10 & Total \\
\hline 1 & 100 & 100 & 80 & 80 & 100 & 100 & 100 & 80 & 40 & 80 & 86 \\
\hline 2 & 80 & 80 & 100 & 100 & 60 & 80 & 60 & 100 & 100 & 60 & 82 \\
\hline 3 & 100 & 60 & 80 & 80 & 100 & 80 & 100 & 100 & 100 & 80 & 88 \\
\hline 4 & 40 & 80 & 80 & 80 & 80 & 100 & 100 & 100 & 80 & 80 & 82 \\
\hline 5 & 100 & 100 & 80 & 80 & 60 & 100 & 80 & 80 & 80 & 60 & 82 \\
\hline 6 & 60 & 60 & 80 & 80 & 80 & 80 & 60 & 80 & 80 & 100 & 76 \\
\hline 7 & 40 & 100 & 80 & 60 & 60 & 100 & 80 & 100 & 100 & 80 & 80 \\
\hline 8 & 80 & 40 & 100 & 80 & 40 & 80 & 100 & 80 & 80 & 100 & 78 \\
\hline 9 & 80 & 100 & 100 & 80 & 100 & 80 & 60 & 60 & 100 & 40 & 80 \\
\hline 10 & 100 & 60 & 80 & 100 & 100 & 80 & 100 & 80 & 80 & 40 & 82 \\
\hline$\overline{A c c}$ & F1 & F2 & F3 & F4 & F5 & F6 & F7 & F8 & F9 & F10 & Total \\
\hline$\overline{1}$ & 80 & 90 & 70 & 60 & 100 & 90 & 100 & 90 & 50 & 80 & 81 \\
\hline 2 & 90 & 60 & 90 & 90 & 60 & 80 & 70 & 100 & 100 & 80 & 82 \\
\hline 3 & 80 & 80 & 70 & 80 & 90 & 80 & 90 & 90 & 100 & 70 & 83 \\
\hline 4 & 60 & 90 & 80 & 90 & 80 & 90 & 80 & 90 & 80 & 80 & 82 \\
\hline 5 & 100 & 90 & 80 & 90 & 80 & 90 & 80 & 70 & 60 & 80 & 82 \\
\hline 6 & 80 & 80 & 90 & 90 & 80 & 80 & 70 & 80 & 90 & 90 & 83 \\
\hline 7 & 50 & 100 & 90 & 80 & 70 & 80 & 70 & 100 & 90 & 90 & 82 \\
\hline 8 & 70 & 60 & 60 & 90 & 70 & 90 & 100 & 90 & 90 & 100 & 82 \\
\hline 9 & 90 & 80 & 80 & 90 & 80 & 90 & 80 & 80 & 80 & 60 & 81 \\
\hline 10 & 90 & 70 & 90 & 80 & 100 & 90 & 100 & 80 & 60 & 50 & 81 \\
\hline
\end{tabular}




\subsection{Statistical Analysis}

The 10 -fold cross validation repeated 10 times, each time the division is randomly generated. The statistical results are listed in Table 1 . The average sensitivity, average specificity, average accuracy are $82.20 \pm 3.94 \%, 81.60 \pm 3.50 \%, 81.90 \pm 0.74 \%$.

\section{Conclusions}

This paper proposed a new method of 'WE-PSOSVM' to identify Ford vehicles. The results showed our method yields a sensitivity of $82.20 \pm 3.94 \%$, a specificity of $81.60 \pm 3.50 \%$, and an accuracy of $81.90 \pm 0.74 \%$. In the future, we shall test advanced variants of SVM, including kernel SVM. We shall also try to use PSOSVM to identify vehicles of other brands.

\section{References}

[1] Patil, P., C. Kanagasabapathi, and S.S. Yellampalli. Automatic number plate recognition system for vehicle identification. in International Conference on Electrical, Electronics, Communication, Computer, and Optimization Techniques (ICEECCOT). 2017. Mysuru, India: IEEE. p. 431-434

[2] Rokonuzzaman, M., et al. Automatic vehicle identification system using machine learning and robot operating system (ROS). in 4th International Conference on Advances in Electrical Engineering (ICAEE). 2017. Dhaka, Bangladesh: IEEE. p. 253-258

[3] Jia, W.-J. and E. Lee. Ford Motor Side-View Recognition System Based on Wavelet Entropy and Back Propagation Neural Network and Levenberg-Marquardt Algorithm. in Eighth International Symposium on Parallel Architectures, Algorithms and Programming (PAAP). 2017. Haikou, China: Springer Singapore. p. 3-12

[4] Jia, W.-J. Ford Motorcar Identification from Single-Camera Side-View Image Based on Convolutional Neural Network. in 18th International Conference on Intelligent Data Engineering and Automated Learning (IDEAL). 2017. Guilin, China: Springer. p. 173-180

[5] Wu, X., Tea category identification based on optimal wavelet entropy and weighted k-Nearest Neighbors algorithm. Multimedia Tools and Applications, 2018. 77(3): p. 3745-3759

[6] Atangana, A., Application of stationary wavelet entropy in pathological brain detection. Multimedia Tools and Applications, 2018. 77(3): p. 3701-3714

[7] Zhou, X., Tea Category Classification Based on Feed-Forward Neural Network and Two-Dimensional Wavelet Entropy, in Third International Conference on High Performance Computing and Applications, J. Xie, et al., Editors. 2016, Springer International Publishing: Cham. p. 48-54.

[8] Phillips, P., Intelligent facial emotion recognition based on stationary wavelet entropy and Jaya algorithm. Neurocomputing, 2018. 272: p. 668-676

[9] Gorriz, J.M., Multivariate approach for Alzheimer's disease detection using stationary wavelet entropy and predator-prey particle swarm optimization. Journal of Alzheimer's Disease, 2017, DOI: 10.3233/JAD-170069.

[10] Bottegal, G. and G. Pillonetto, The generalized cross validation filter. Automatica, 2018. 90: p. 130-137

[11] Wang, S.-H., Single slice based detection for Alzheimer's disease via wavelet entropy and multilayer perceptron trained by biogeography-based optimization. Multimedia Tools and Applications, 2016, DOI: 10.1007/s11042-016-4222-4.

[12] Nayak, D.R., Detection of unilateral hearing loss by Stationary Wavelet Entropy. CNS \& Neurological Disorders - Drug Targets, 2017. 16(2): p. 15-24 
[13] Lu, H.M., Facial Emotion Recognition Based on Biorthogonal Wavelet Entropy, Fuzzy Support Vector Machine, and Stratified Cross Validation. IEEE Access, 2016. 4: p. 8375-8385

[14] Gorriz, J.M. and J. Ramírez, Wavelet entropy and directed acyclic graph support vector machine for detection of patients with unilateral hearing loss in MRI scanning. Frontiers in Computational Neuroscience, 2016. 10: Article ID. 160

[15] Zhou, X.-X., Comparison of machine learning methods for stationary wavelet entropy-based multiple sclerosis detection: decision tree, k-nearest neighbors, and support vector machine. Simulation, 2016. 92(9): p. 861-871

[16] Zhou, X.X. and G.S. Zhang, Detection of abnormal MR brains based on wavelet entropy and feature selection. IEEJ Transactions on Electrical and Electronic Engineering, 2016. 11(3): p. 364-373

[17] Sun, P., Pathological brain detection based on wavelet entropy and Hu moment invariants. Bio-Medical Materials and Engineering, 2015. 26(s1): p. 1283-1290

[18] Rizal, A., R. Hidayat, and H.A. Nugroho. Multilevel wavelet packet entropy: A new strategy for lung sound feature extraction based on wavelet entropy. in International Conference on Robotics, Automation and Sciences (ICORAS). 2017. Melaka, Malaysia: IEEE. p. 1-5

[19] Phillips, P., Z. Dong, and J. Yang, Pathological brain detection in magnetic resonance imaging scanning by wavelet entropy and hybridization of biogeography-based optimization and particle swarm optimization. Progress In Electromagnetics Research, 2015. 152: p. 41-58

[20] Zhan, T.M. and Y. Chen, Multiple Sclerosis Detection Based on Biorthogonal Wavelet Transform, RBF Kernel Principal Component Analysis, and Logistic Regression. IEEE Access, 2016. 4: p. 7567-7576

[21] Chen, Y. and H. Lu, Wavelet energy entropy and linear regression classifier for detecting abnormal breasts. Multimedia Tools and Applications, 2018. 77(3): p. 3813-3832

[22] Chen, Y. and X.-Q. Chen, Sensorineural hearing loss detection via discrete wavelet transform and principal component analysis combined with generalized eigenvalue proximal support vector machine and Tikhonov regularization. Multimedia Tools and Applications, 2016. 77(3): p. 3775-3793

[23] Zhou, X.-X., et al., Combination of stationary wavelet transform and kernel support vector machines for pathological brain detection. Simulation, 2016. 92(9): p. 827-837

[24] Phoemphon, S., C. So-In, and D. Niyato, A hybrid model using fuzzy logic and an extreme learning machine with vector particle swarm optimization for wireless sensor network localization. Applied Soft Computing, 2018. 65: p. 101-120

[25] Hou, X.-X., Alcoholism detection by medical robots based on $\mathrm{Hu}$ moment invariants and predator-prey adaptive-inertia chaotic particle swarm optimization. Computers and Electrical Engineering, 2017. 63: p. 126-138

[26] Yang, J.F. and P. Sun, Magnetic resonance brain classification by a novel binary particle swarm optimization with mutation and time-varying acceleration coefficients. Biomedical Engineering-Biomedizinische Technik, 2016. 61(4): p. 431-441

[27] Ji, G., A comprehensive survey on particle swarm optimization algorithm and its applications. Mathematical Problems in Engineering, 2015: Article ID. 931256

[28] Ji, G., An MR brain images classifier system via particle swarm optimization and kernel support vector machine. The Scientific World Journal, 2013: Article ID. 130134

[29] Wu, L., UCAV path planning by fitness-scaling adaptive chaotic particle swarm optimization. Mathematical Problems in Engineering, 2013: Article ID. 705238 
[30] Wu, L., Crop Classification by forward neural network with adaptive chaotic particle swarm optimization. Sensors, 2011. 11(5): p. 4721-4743

[31] Li, P. and G. Liu, Pathological Brain Detection via Wavelet Packet Tsallis Entropy and Real-Coded Biogeography-based Optimization. Fundamenta Informaticae, 2017. 151(1-4): p. 275-291

[32] Wu, X., Smart detection on abnormal breasts in digital mammography based on contrast-limited adaptive histogram equalization and chaotic adaptive real-coded biogeography-based optimization. Simulation, 2016. 92(9): p. 873-885

[33] Wu, J., Fruit classification by biogeography-based optimization and feedforward neural network. Expert Systems, 2016. 33(3): p. 239-253

[34] Wei, L., Fruit classification by wavelet-entropy and feedforward neural network trained by fitness-scaled chaotic ABC and biogeography-based optimization. Entropy, 2015. 17(8): p. 5711-5728

[35] Oguntunde, P.G., G. Lischeid, and O. Dietrich, Relationship between rice yield and climate variables in southwest Nigeria using multiple linear regression and support vector machine analysis. International Journal of Biometeorology, 2018. 62(3): p. 459-469

[36] Chen, Y. Pathological Brain Detection by Wavelet-Energy and Fuzzy Support Vector Machine. in 8th International Symposium on Computational Intelligence and Design (ISCID). 2015. Hangzhou, China: IEEE. p. 409-412

[37] Chen, M., Morphological analysis of dendrites and spines by hybridization of ridge detection with twin support vector machine. PeerJ, 2016. 4: Article ID. e2207

[38] Yang, M., Dual-Tree Complex Wavelet Transform and Twin Support Vector Machine for Pathological Brain Detection. Applied Sciences, 2016. 6(6): Article ID. 169 